\title{
On numerical approximation of incompresible fluid flow with free surface influenced by surface tension
}

\author{
Petr Sváček ${ }^{\mathrm{a}}$ \\ Czech Technical University in Prague, Faculty of Mechanical Engineering, Dep. of Technical Mathematics, Karlovo nám. 13, \\ Praha 2, Czech Republic
}

\begin{abstract}
This paper deals with the numerical approximation of two immiscible incompressible fluids flow. The two-dimensional problem is mathematically described with the aid of the incompressible Navier-Stokes equations. The motion of the two phases is treated with the aid of level set method. The surface tension effects are taken into account as well as the contact angle influence. The problem is formulated in a weak sense and discretized with the aid of the finite element method. The details of the numerical methods are given and several possibilities of the realization of the surface tension term are discussed. Numerical results are shown.
\end{abstract}

\section{Introduction}

The numerical approximation of two-phase flows with free surface is very important in various scientific as well as in technical applications or industrial processes, see e.g. [1], [2], [3]. The kinematics and dynamics of the fluid interface plays an important role in many of these processes. The approximation of free surface flow influenced by the high surface tension and possibly contact angles is very difficult problem, see e.g. [4], [5], [6], [7], [8].

In order to approximate free surface flows there are several fast, robust and accurate numerical methods available dealing with fixed computing grids. Particularly, these methods are the marker and cell method [9], volume of fluid method [10] and level set method [11], [12], [13]. There are many papers describing the application of the mentioned methods, but its reliability is usually not tested.

In this paper, the two-dimensional flow of two immiscible fluids is considered, mathematically described. In order to take into account the surface tension effects the variational formulation is introduced and the discretization with the the finite element method is applied. The motion of the interface between these two fluids (free surface) is realized using the level set method, cf. [11]. A modification of the standard finite element method is used in order to properly address the arising spurious currents nearby the free surface caused by the discontinuous pressure, see [7] or [5]. The implemented method is verified by solution of a benchmark problem, cf. [4].

\section{Mathematical model}

In this section two immiscible fluids are assumed to occupy a domain $\Omega \subset \mathbb{R}^{2}$. The domain $\Omega$ is a bounded com-

\footnotetext{
${ }^{a}$ Corresponding author: petr.svacek@fs.cvut.cz
}

putational domain with the Lipschitz continuous boundary decomposed into three mutually disjoint parts $\Gamma_{W}$ (representing fixed wall), $\Gamma_{S}$ (representing part of boundary where the slip boundary condition is prescribed) and $\Gamma_{O}$ (outlet part of the boundary). Each fluid is assumed to occupy a time dependent subdomain $\left(\Omega_{(t)}^{1}\right.$ or $\left.\Omega_{(t)}^{2}\right)$ with the moving interface $\hat{\Gamma}_{t}$ (free surface), i.e. the interface is the common boundary of both subdomains $\hat{\Gamma}_{t}=\partial \Omega_{(t)}^{1} \cap \partial \Omega_{(t)}^{2}$. Both incompressible fluids are characterized by the density $\left(\rho_{1}\right.$ and $\left.\rho_{2}\right)$ and its viscosity $\left(\mu_{1}\right.$ and $\mu_{2}$ ).

In order to describe the mathematical model in a single equation, the Heaviside function $H(x, t)$ is introduced

$$
H(x, t)=\left\{\begin{aligned}
1 & \text { for } x \in \Omega_{(t)}^{1}, \\
\frac{1}{2} & \text { for } x \in \hat{\Gamma}_{t}, \\
0 & \text { for } x \in \Omega_{(t)}^{2},
\end{aligned}\right.
$$

Further, the density $\rho(x, t)$ and viscosity $\mu(x, t)$ functions are defined using the Heaviside functions as functions giving either the fluid local density or viscosity at the point $x \in \Omega$ at time $t \in(0, T)$. Thus the density function reads

$$
\rho(x, t)=\rho_{1} H(x, t)+(1-H(x, t)) \rho_{2},
$$

and the viscosity function is given by formula

$$
\mu(x, t)=\mu_{1} H(x, t)+(1-H(x, t)) \mu_{2} .
$$

Similarly, the pressure and the flow velocity functions defined on the domain $\Omega$ are denoted by $p(x, t)$ and $\boldsymbol{u}=$ $\boldsymbol{u}(x, t)$, respectively. The flow motion in $\Omega_{(t)}^{i}, i=1,2$ is then described by the incompressible Navier-Stokes equations

$$
\rho \frac{\partial \boldsymbol{u}}{\partial t}+\rho(\boldsymbol{u} \cdot \nabla) \boldsymbol{u}-\nabla \cdot \boldsymbol{\sigma}=\rho \boldsymbol{f} .
$$




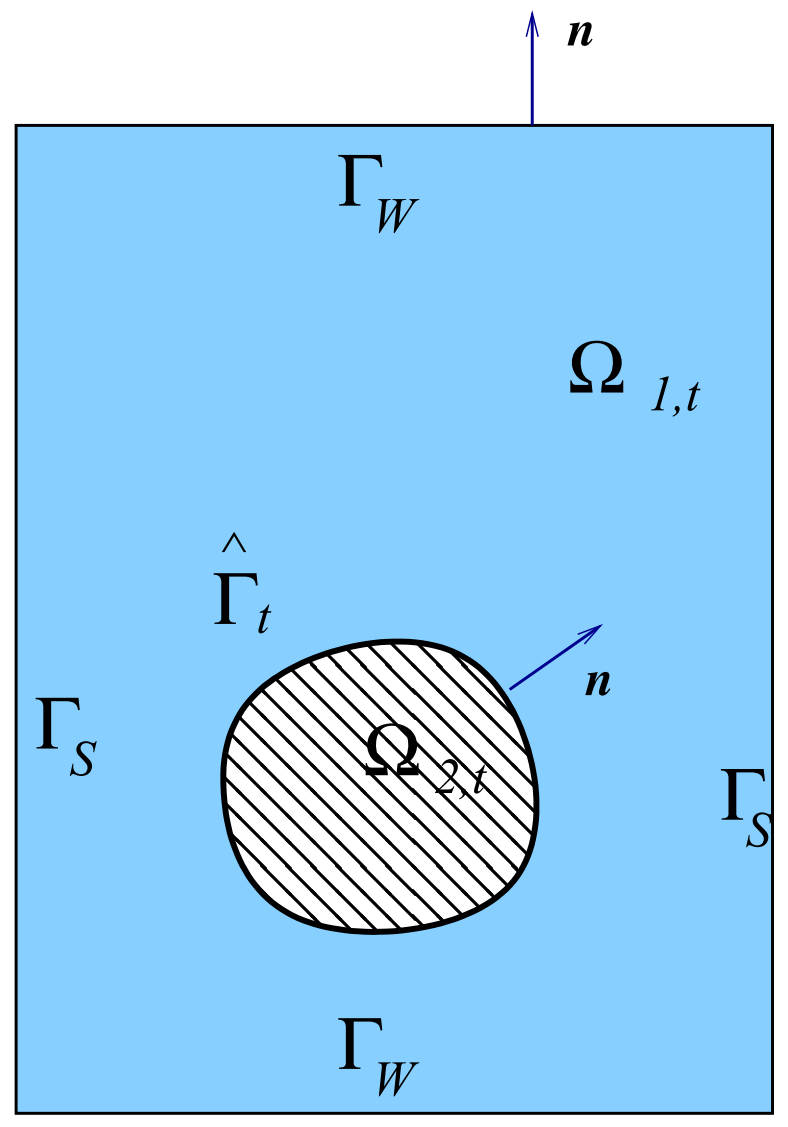

Fig. 1. Sketch of the computational domain.

On the interface $\hat{\Gamma}_{t}$ the following boundary conditions including the surface tension effects needs to be specified

$$
\begin{array}{ll}
\text { a) } & \left.\boldsymbol{u}\right|_{\Omega_{(t)}^{1}}=\left.\boldsymbol{u}\right|_{\Omega_{(t)}^{2}} \\
b) & {[\boldsymbol{\sigma} \cdot \boldsymbol{n}]=\gamma \kappa \mathbf{n},}
\end{array}
$$

where $\gamma$ is the surface tension coefficient, $\kappa$ denotes the curvature of the interface $\Gamma_{I}$ and $\boldsymbol{n}$ here denotes the normal to the $\Gamma_{I}$ pointing into $\Omega_{(t)}^{2}$.

The Navier-Stokes equations (4) together with the interface condition (5) can be written in $\Omega=\Omega_{(t)}^{1} \cup \hat{\Gamma}_{t} \cup \Omega_{(t)}^{2}$ as

$$
\rho \frac{\partial \boldsymbol{u}}{\partial t}+\rho(\boldsymbol{u} \cdot \nabla) \boldsymbol{u}-\nabla \cdot \boldsymbol{\sigma}=\rho \boldsymbol{f}+\gamma \kappa \boldsymbol{n} \delta_{\hat{\Gamma}_{t}},
$$

where $\delta_{\hat{\Gamma}_{t}}$ is the delta function of the interface $\hat{\Gamma}_{t}$ and $\boldsymbol{n}$ is the unit normal vector to the interface $\hat{\Gamma}_{t}$ (oriented into $\left.\Omega_{(t)}^{2}\right)$. The meaning of Eq. (6) is clear in the weak formulation: we seek $\boldsymbol{u}$ such that for any $\boldsymbol{v} \in \boldsymbol{H}^{1}(\Omega)$ holds

$$
\begin{array}{r}
\int_{\Omega} \rho\left(\frac{\partial \boldsymbol{u}}{\partial t}+(\boldsymbol{u} \cdot \nabla) \boldsymbol{u}\right) \cdot \boldsymbol{v}+\boldsymbol{\sigma} \cdot(\nabla \boldsymbol{v}) \mathrm{d} x= \\
\int_{\hat{\Gamma}_{t}} \gamma \kappa \boldsymbol{n} \cdot \boldsymbol{v} \mathrm{d} S+\int_{\Omega} \rho \boldsymbol{f} \cdot \boldsymbol{v} \mathrm{d} x .
\end{array}
$$

Furthermore, system (6) is equipped with an initial condition $\boldsymbol{u}=0$ and with suitable boundary conditions.
Further, on the boundary $\partial \Omega$ the following boundary conditions are prescribed
a) $\boldsymbol{u}=0$
on $\Gamma_{W}$,
b) $\boldsymbol{u} \cdot \boldsymbol{n}=0$
$\boldsymbol{t} \cdot(\boldsymbol{\sigma} \cdot \boldsymbol{n})=0$
on $\Gamma_{S}$,
c) $\boldsymbol{\sigma} \cdot \boldsymbol{n}=0$
on $\Gamma_{O}$.

Level set method. The free surface motion is numerically treated with the aid of the level set method, see e.g. [11]. The level set function generally can be considered as the signed distance function being zero on the interface $\hat{\Gamma}_{t}$ at any time instant $t$, positive in $\Omega_{(t)}^{1}$ and negative in $\Omega_{(t)}^{2}$. In practical realization the level set function is used only to implicitly define the interface $\hat{\Gamma}_{t}$ as the set of points satisfying the condition $\phi(x, t)=0$. With the aid of a level set function $\phi(x, t)$ the Heaviside function $H(x, t)$ satisfy

$$
H(x, t)=\left\{\begin{aligned}
1 & \text { for } \phi(x, t)>0 \\
\frac{1}{2} & \text { for } \phi(x, t)=0 \\
0 & \text { for } \phi(x, t)<0 .
\end{aligned}\right.
$$

The motion of the interface $\hat{\Gamma}_{t}$ is then realized by solving the transport equation for the function $\phi(x, t)$, i.e.

$$
\frac{\partial \phi}{\partial t}+\boldsymbol{u} \cdot \nabla \phi=0
$$

Equation (9) means that the interface $\hat{\Gamma}_{t}$ moves in time with the flow velocity $\boldsymbol{u}$.

Surface tension. The surface tension realized with the aid of the weak reformulation, see [4], [14]. To this end the tangent derivative $\nabla_{\Gamma}$ is defined as

$$
\nabla_{\Gamma} g=\nabla g-(\boldsymbol{n} \cdot \nabla g) \boldsymbol{n}
$$

and similarly the second order tangent derivative, i.e. the Laplace-Beltrami operator, (see [14]) as

$$
\triangle_{\Gamma}=\nabla_{\Gamma} \cdot \nabla_{\Gamma}
$$

Now, using the relation

$$
\kappa \boldsymbol{n}=\triangle_{\Gamma} x,
$$

and applying the integration by parts on $\hat{\Gamma}_{t}$ we get

$\int_{\hat{\Gamma}_{t}} \gamma \kappa \boldsymbol{n} \cdot \boldsymbol{v} \mathrm{d} S=\int_{\partial \hat{\Gamma}_{t}} \gamma\left(\nabla_{\Gamma} x\right) \cdot \boldsymbol{v}-\int_{\hat{\Gamma}_{t}} \gamma\left(\nabla_{\Gamma} x\right) \cdot\left(\nabla_{\Gamma} \boldsymbol{v}\right) \mathrm{d} S$,

where on the right hand side the integral over $\partial \hat{\Gamma}_{t}$ denotes the integral over the contact lines (in 3D). For the twodimensional problem the integral over $\partial \hat{\Gamma}_{t}$ is the sum over the contact points, which - in the case of $\hat{\Gamma}_{t}$ being a closed curve - is the empty set and equals zero. In the other case this reformulation naturally includes the contact angles into the weak formulation of the problem. 


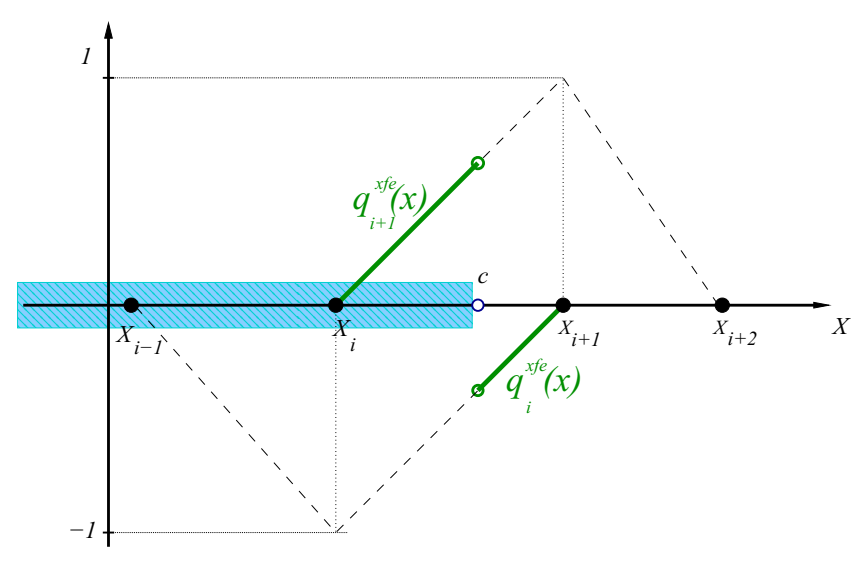

Fig. 2. The base functions of the extended finite element space shown for $1 \mathrm{D}$ case on the grid given by points $x_{i-2}$, $x_{i-1}, x_{i}$ and $x_{i+1}$. The interface at time $t$ is located between the nodes $x_{i}$ and $x_{i+1}$ at $x=c$ and the interval where the levelset function is positive $(\phi(x, t)>0)$ is marked by the dashed part of the $\mathrm{x}$-axis.

\section{Numerical approximation}

Flow step. In order to discritize the problem in time an equidistant division $t_{n}=n \Delta t$ of the time interval $[0, T)$ is considered with a constant time step $\Delta t=T / N$. Furthermore, the approximations of the flow velocity $\boldsymbol{u}$, the presure $p$, the density $\rho$ and the viscosity $\mu$ at the time instant $t_{n}$ are denoted by $\boldsymbol{u}^{(n)}, p^{(n)}, \phi^{(n)}, \rho^{n}$ and $\mu^{n}$, respectively. The time derivatives are then approximated by the second order backward difference formula as

$$
\begin{aligned}
&\left.\frac{\partial \boldsymbol{u}}{\partial t}\right|_{t=t_{n+1}} \approx \frac{3 \boldsymbol{u}^{(n+1)}-4 \boldsymbol{u}^{(n)}+\boldsymbol{u}^{(n-1)}}{\Delta t}, \\
&\left.\frac{\partial \phi}{\partial t}\right|_{t=t_{n+1}} \approx \frac{3 \phi^{(n+1)}-4 \phi^{(n)}+\phi^{(n-1)}}{\Delta t} .
\end{aligned}
$$

The time discretized weak formulation of Eq. (6) then reads: Find $\boldsymbol{u}=\boldsymbol{u}^{n+1} \in \boldsymbol{V}$ and $p=p^{n+1} \in Q$ such that

$$
\begin{aligned}
& \int_{\Omega} \rho^{n+1}(x)\left(\frac{\boldsymbol{u}-\boldsymbol{u}^{n}}{\Delta t}+(\boldsymbol{u} \cdot \nabla) \boldsymbol{u}\right) \cdot \boldsymbol{v} \mathrm{d} x \\
& +\int_{\Omega} \mu^{n+1}(x) \nabla \boldsymbol{u} \cdot \nabla \boldsymbol{v}-p(\nabla \cdot \boldsymbol{v}) \mathrm{d} x+\int_{\Omega}(\nabla \cdot \boldsymbol{u}) q \mathrm{~d} x \\
& =-\int_{\hat{\Gamma}^{n+1}} \gamma\left(\nabla_{\Gamma} x\right) \cdot\left(\nabla_{\Gamma} \boldsymbol{v}\right) \mathrm{d} S+\int_{\Omega} \rho^{n+1}(x) \boldsymbol{f} \cdot \boldsymbol{v} \mathrm{d} x
\end{aligned}
$$

holds for all $\boldsymbol{v} \in \boldsymbol{V}$ and $q \in Q$.

In the practical computations the polygonal computational domain $\Omega$ is used and the spaces $\boldsymbol{V}$ and $Q$ are approximated by the FE subspaces $\boldsymbol{V}_{h}$ and $Q_{h}$, respectively, defined over an admissible triangulation $\mathcal{T}_{h}$ of the domain $\Omega$. The Taylor-Hood finite elements are used for approximation of velocity and pressure, i.e. the velocity components are sought in the space $\boldsymbol{V}_{h}=\left[H_{h}\right]^{2} \subset \boldsymbol{V}$, where the space $H_{h}$ is defined by

$$
H_{h}=\left\{\phi \in C(\bar{\Omega}) ;\left.\phi\right|_{K} \in P_{2}(K) \text { for each } K \in \mathcal{T}_{h}\right\} \text {, }
$$

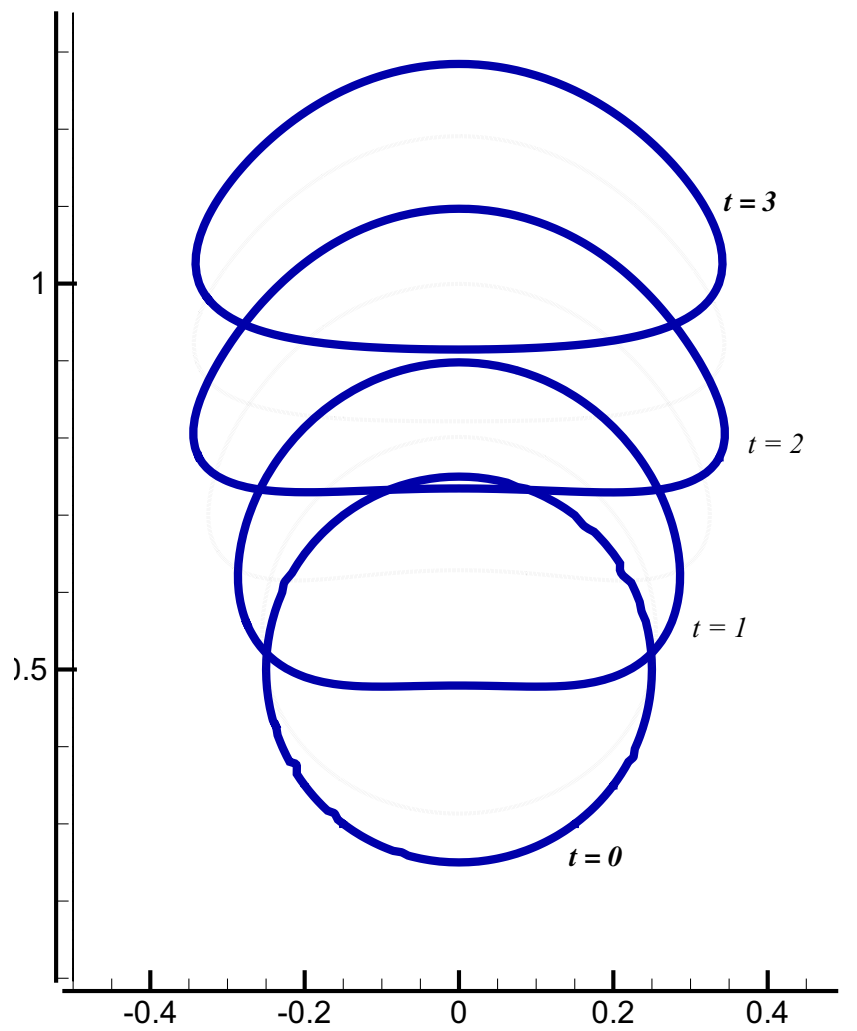

Fig. 3. The result of the rising bubble test for the first test case. The shape of the interface is shown at the time instants $t \in\{0,0.5,1,1.5,2,2.5,3\}$. The effect of much higher surface tension leads to a development of a stable bubble shape.

and $P_{k}(K)$ denotes the space of all polynomials of degree less or equal to $k$ on $K$. Further, the pressure (as well as the level set function) is approximated in the space

$$
Q_{h}=\left\{\phi \in C(\bar{\Omega}):\left.\phi\right|_{K} \in P_{1}(K) \text { for each } K \in \mathcal{T}_{h}\right\}
$$

The discrete flow problem then reads: Find $\boldsymbol{u}_{h}=$ $\boldsymbol{u}_{h}^{n+1} \in \boldsymbol{V}_{h}$ and $p_{h}=p_{h}^{n+1}$ such that Eq. (11) holds for any test function $\boldsymbol{v}:=\boldsymbol{v}_{h} \in \boldsymbol{V}_{h}$ and $q:=q_{h} \in Q_{h}$. In order to treat the discontinuity of the pressure due to the presence of the surface tension the extended finite element method (XFEM) is applied, see e.g. [7].

Extended finite element method. The XFEM method is used to treat the possible numerical instability caused by the surface tension: the pressure is discontinuous along the interface due to the applied surface tension term. The numerical approximation with the aid of Taylor-Hood finite element means that the presure is continous. In order to capture the pressure discontinuity the pressure finite element space $Q_{h}$ is enriched using the localization of a enrichment function, in this case the enrichment of the Heaviside function $H_{\Gamma}(x)$ given as the Heaviside function $H_{\Gamma}(x)=H\left(x, t_{n+1}\right)$ at the time instant $t_{n+1}$.

In order to enrich the finite element space, the original base functions of $Q_{h}$ are used, i.e. we denote the index 
set $\mathcal{J}=\{1, \ldots, n\}, n=\operatorname{dim} Q_{h}$ and the mesh nodes by $x_{j}, j \in \mathcal{J}$. The nodal base functions are then denoted by $q_{i} \in Q_{h}, i \in \mathcal{J}$ and satisfy $q_{i}\left(x_{j}\right)=\delta_{i j}$. The $\mathcal{J}^{\prime}$ is the subset of all the neighbours of the interface $\hat{\Gamma}_{t}$, i.e.

$$
\mathcal{J}^{\prime}=\left\{j \in \mathcal{J}: \operatorname{supp} q_{j} \cap \hat{\Gamma}_{t} \neq \emptyset\right\} .
$$

Finally, the enrichment of the space $Q_{h}$ is made using the discontinuous base functions $q_{j}^{x f e m}$ defined by

$$
q_{j}^{x f e m}(x)=q_{j}(x)\left(H_{\Gamma}(x)-H_{\Gamma}\left(x_{j}\right)\right),
$$

where the term $-q_{j}(x) H_{\Gamma}\left(x_{j}\right)$ makes the support of the function $q_{j}^{x f e m}(x)$ localized to the single element containing the interface, see Fig. 2.

Here, $H_{\Gamma}\left(x_{j}\right)$ can be left out from the right hand side as this only adds a constant multiple of the continuous base function $q_{j}(x)$. On the other hand, this term makes the function $q_{j}^{x f e m}(x)$ being zero at every node $x_{i}, i \in \mathcal{J}$ and also makes the support of $q_{j}^{x f e m}(x)$ localized only to the elements containing the interface $\hat{\Gamma}_{t}$, which simplifies the practical discretization of the problem. The FE space $Q_{h}$ is then replaced by the extended FE space

$$
Q_{h}^{x f e m}=Q_{h} \oplus \operatorname{span}\left\{q_{j}^{x f e m}: j \in \mathcal{J}^{\prime}\right\} .
$$

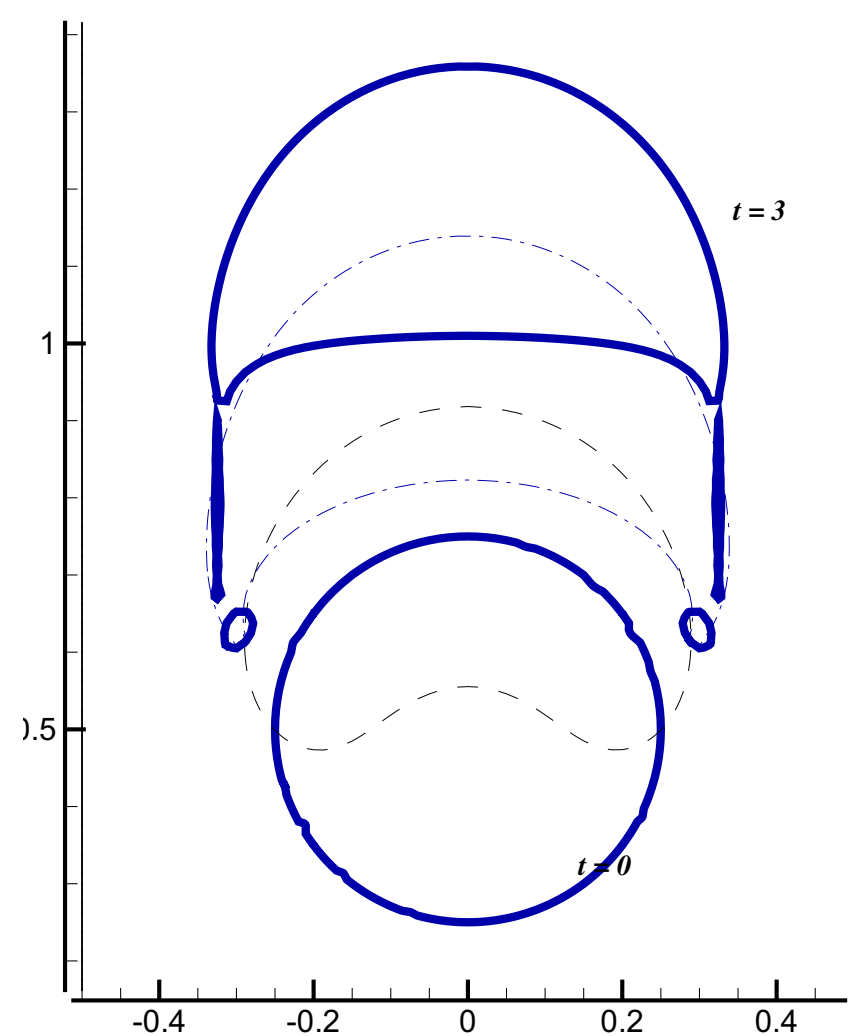

Fig. 4. The result of the rising bubble test for the second test case. The shape of the interface is shown at the time instants $t \in\{0,1,2,3\}$.
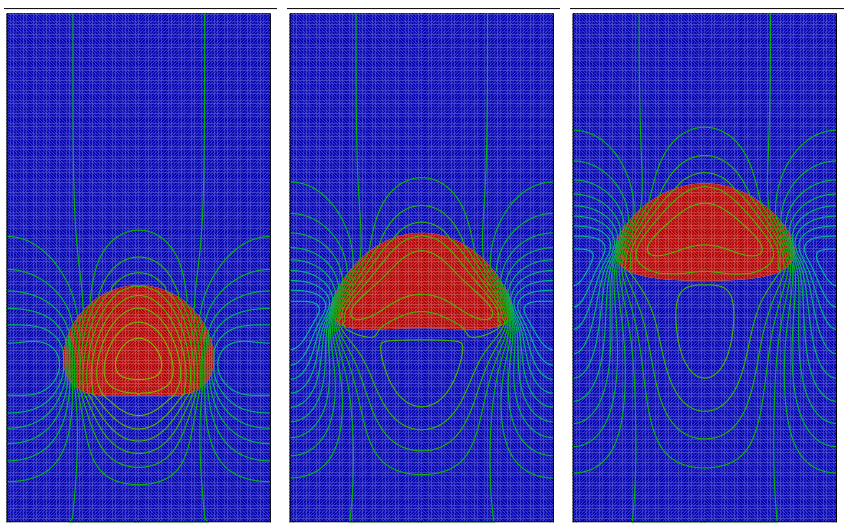

Fig. 5. The result of the rising bubble case I: The shape of the interface and the isolines of the vertical component of the flow velocity at time instants $t \in\{1,2,3\}$ computed at the grid with $h=1 / 80$.
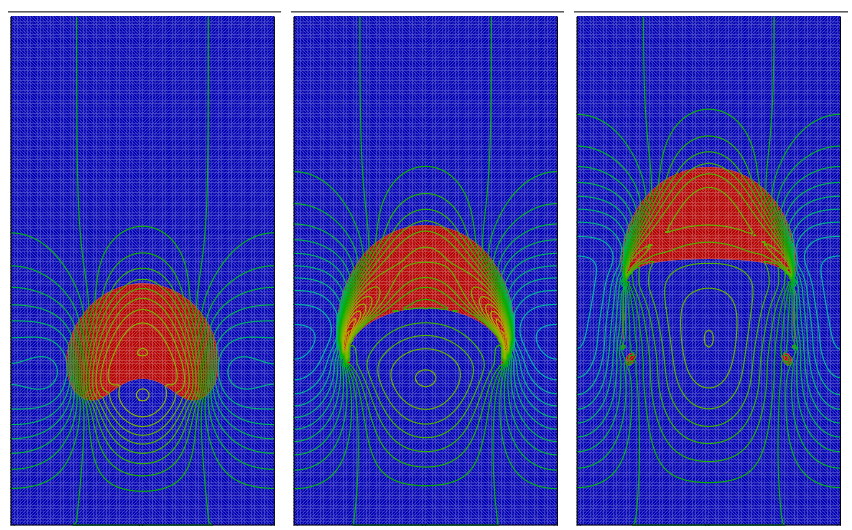

Fig. 6. The result of the rising bubble case II: The shape of the interface and the isolines of the vertical component of the flow velocity at time instants $t \in\{1,2,3\}$ at the finest grid with the spatial step $h=1 / 80$.

Level set step and coupled problem. The level set equation (9) is numerically approximated with the aid of finite element method. In order to get the numerically stability of the scheme, the algebraic flux corrections are applied, see [15]. Moreover, in order to keep the level set function as the signed distance function, a re-initialization step is applied, see also [4]. The solution of the coupled problem is then performed by a de-coupled algorithm with strong coupling. This means, that both problems are solved several times in order to get a convergence criteria satisfied. In practical computations the level set step is solved first followed by solution of equation (11) for approximation of the flow velocity $\boldsymbol{u}^{n+1}$ and the pressure $p^{n+1}$.

\section{Numerical results}

In order to verify the described numerical method, the two benchmarks for the case of a rising bubble considered in [4] were numerically approximated. The first test case (case I) used the following values of the fluid's densities 
$\rho_{1}=1000 \mathrm{~kg} \mathrm{~m}^{-3}, \rho_{2}=100 \mathrm{~kg} \mathrm{~m}^{-3}$, the fluid's viscosities $\mu_{1}=10 \mathrm{Pas}, \mu_{2}=1 \mathrm{Pas}$, the volume force represented by the gravitational acceleration $\boldsymbol{f}=(0,-0.98) \mathrm{ms}^{-2}$ and the surface tension $\gamma=24.5 \mathrm{~N} / \mathrm{m}$. The height of the considered computational domain was chosen as $H=$ $2 \mathrm{~m}$ and the width $W$ was equal to $1 \mathrm{~m}$. The fluid 2 was originally located inside of the circle with the diameter of $0.5 \mathrm{~m}$ with its center located $0.5 \mathrm{~m}$ up from the bottom of the domain, see 1 with the boundary $\Gamma_{W}$ being the bottom and top of the domain, and the side (left and right) boundaries were chosen as $\Gamma_{S}$ (i.e. $\Gamma_{O}=\emptyset$ ). Due to the gravitational force, the second fluid with the lower density starts to rise and deform its shape, see Fig. 3 However, due to high surface tension a stable shape is developed after a short time period. Since this time the fluid keeps rising in an undeformed shape, see Fig. 5.

Following [4] also the second benchmark test was approximated (test case II). In this case the fluid's densities were $\rho_{1}=1000 \mathrm{~kg} \mathrm{~m}^{-3}$ and $\rho_{2}=1 \mathrm{~kg} \mathrm{~m}^{-3}$, fluid's viscosities were $\mu_{1}=10 \mathrm{Pas}$ and $\mu_{2}=0.1 \mathrm{~Pa}$. The same gravitational acceleration was used $\boldsymbol{f}=(0,-0.98) \mathrm{m} \mathrm{s}^{-2}$ but lower surface tenstion $\gamma=1.96 \mathrm{~N} / \mathrm{m}$ was considered. The development of the free surface is shown in Fig. 4. The development of the shape as well as the vertical component of the flow velocity is shown in Fig. 6 .

The computations were performed on triangular meshes with the equidistant partition. The spatial steps $h=$ $1 / 20,1 / 40$ or $1 / 80$ were used. The step $h=1 / 40$ was the coarsest mesh used in [4]. The time step used in the computation was $\Delta t=0.002$. The motion of the domain $\Omega_{(t)}^{2}$ with the area $\mathcal{A}(t)$ was tracked in terms of the $y$-coordinate of the center of mass

$$
T_{y}(t)=\int_{\Omega_{(t)}^{2}} x_{2} \mathrm{~d} x / \mathcal{A}(t),
$$

the circularity defined by

$$
C(t)=2 \sqrt{\pi \mathcal{A}(t)} / \int_{\partial \Omega_{(t)}^{2}} 1 \mathrm{~d} S
$$

and the rise velocity

$$
V=\int_{\Omega_{(t)}^{2}} u_{2} \mathrm{~d} x / \mathcal{A}(t) .
$$

In order to verify the presented numerical method the values of $T_{y}, C$ and $V$ were computed at every time instant for both the test cases. The graphs of $T_{y}, C$ and $V$ in dependence on time shown in Fig. 7 for the test case I agrees well with the results in [4]. The computations were performed on three grids and the differences were not significant. Similarly, the graphs of $T_{y}, C$ and $V$ in dependence on time shown in Fig. 8 for the test case II is similar to the results presented in [4]. Nevertheless for the benchmark II the results are much more sensitive (particularly for the circularity) to the mesh refinement. See also the quantitative comparison for both cases in Tables 1,2 .
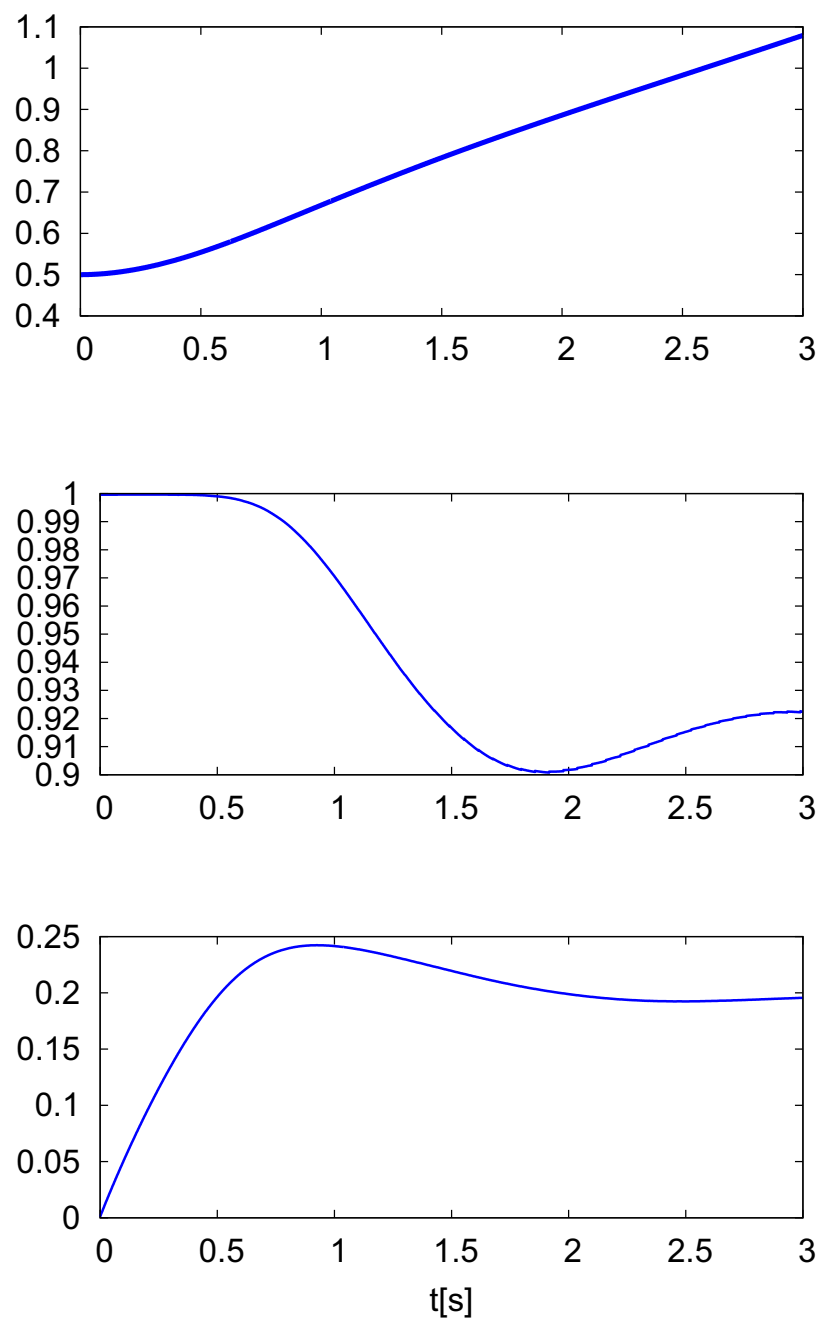

Fig. 7. The quantitative results for the rising bubble case I: The graphs of the center of mass $T_{y}$ (top), the circularity $C$ (middle) and the rise velocity $V$ (bottom) in dependence on time.

Table 1. The quantitative results for the rising bubble case I: the comparison of the computed and the reference quantities.

\begin{tabular}{|l|r|r|}
\hline & ref. [4], case I & present study, case I \\
\hline$T_{y}(3)$ & 1.0813 & 1.0801 \\
$C_{\min }$ & 0.9013 & 0.9025 \\
$t_{C_{\min }}$ & 1.9041 & 1.898 \\
$V_{\max }$ & 0.2417 & 0.2421 \\
$t_{V_{\max }}$ & 0.9213 & 0.92 \\
\hline
\end{tabular}

\section{Conclusion}

The motion of two immiscible fluid was mathematically modelled with the aid of a variational formulation which includes the surface tension and possibly also the contact angles effects. The time discretization of the problem was performed with the aid of the second order backward difference formula. The spatial discretization was done with the aid of the finite element method, where the XFEM was applied in order to capture the disconti- 

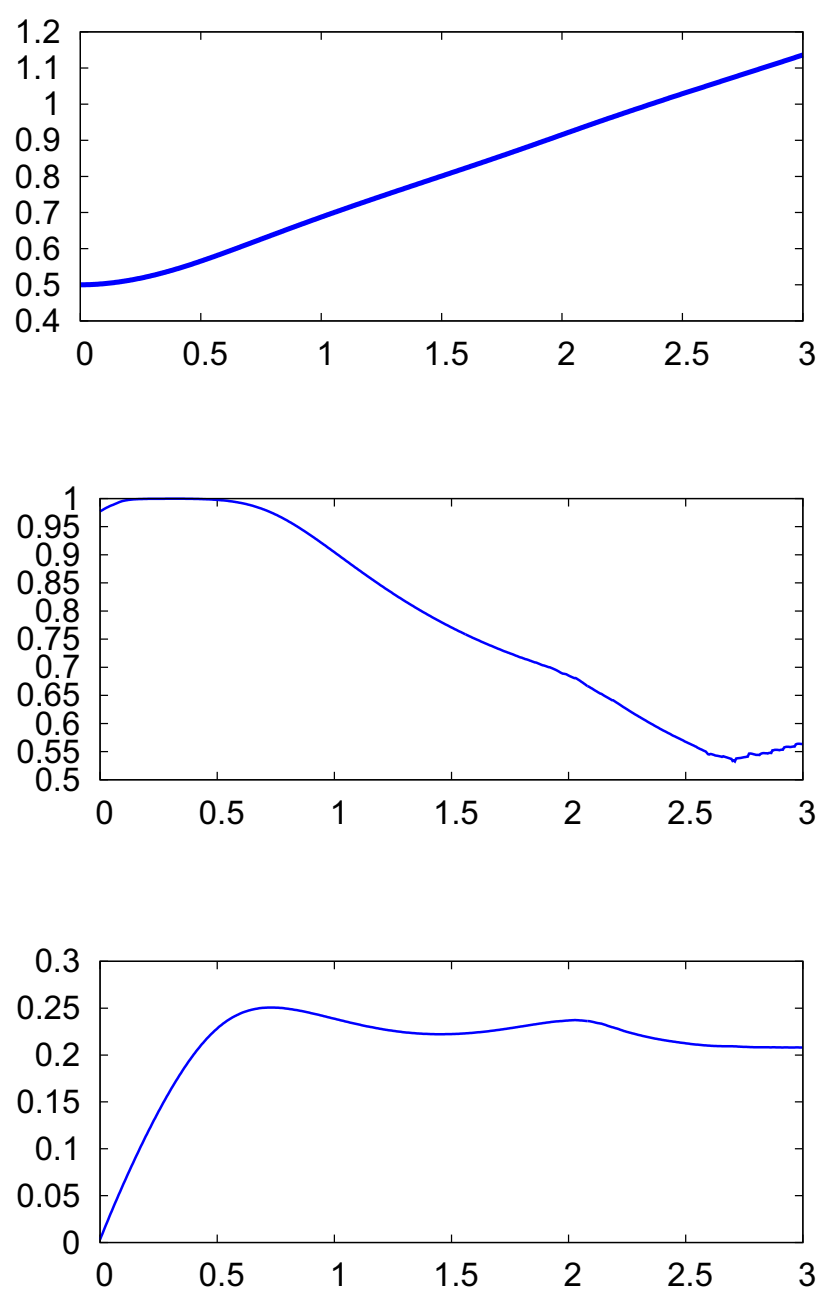

Fig. 8. The quantitative results for the rising bubble case II: The graphs of the center of mass $T_{y}$ (top), the circularity $C$ (middle) and the rise velocity $V$ (bottom) in dependence on time.

Table 2. The quantitative results for the rising bubble case II: the comparison of the computed and the reference quantities.

\begin{tabular}{|l|r|r|}
\hline & ref. [4], case II & present study, case II \\
\hline$T_{y}(3)$ & 1.1380 & 1.13618 \\
$C_{\min }$ & 0.5869 & 0.53 \\
$t_{C_{\min }}$ & 2.4 & 2.7 \\
$V_{\max }$ & 0.2524 & 0.2507 \\
$t_{V_{\max }}$ & 0.7332 & 0.7298 \\
$V_{\max , 2}$ & 0.2434 & 0.237 \\
$t_{V_{\max , 2}}$ & 2.0705 & 2.03 \\
\hline
\end{tabular}

nuity of the pressure along the free surface. The motion of the free surface was treated with the aid of the level set method. The presented numerical method was tested on two benchmark problems from [4] and the obtained numerical results were compared to the reference values.
Acknowledgment This work was supported by the Czech Science Foundation under the Grant No. 13-00522S

\section{References}

1. A. Maiwald, P. Scheller, C. Brücker, R. Schwarze, Flowinduced emulsification of mold powder slag into liquid steel, in STEELSIM 2009 3rd International Conference Simulation and Modelling of Metallurgical Processes in Steelmaking (Leoben, 2009), pp. $162-167$

2. A. Bansal, P. Chapelle, Y. Delannoy, E. Waz, P. Le Brun, J.P. Bellot, Metallurgical and Materials Transactions B 46, 2096 (2015)

3. A.V. Minakov, M.V. Pervukhin, D.V. Platonov, M.Y. Khatsayuk, Computational Mathematics and Mathematical Physics 55, 2066 (2015)

4. S. Hysing, S. Turek, D. Kuzmin, N. Parolini, E. Burman, S. Ganesan, L. Tobiska, International Journal for Numerical Methods in Fluids 60, 1259 (2009)

5. J.W. Barrett, H. Garcke, R. Nürnberg, Computer Methods in Applied Mechanics and Engineering 267, 511 (2013)

6. S.R. Ransau, Tech. Rep. 3/2002, Norwegian University of Science and Technology, Trondheim (2002)

7. H. Sauerland, T.P. Fries, Computers \& Fluids 87, 41 (2013)

8. E. Bänsch, S. Weller, A comparison of several time discretization methods for free surface flows, In ALGORITMY 2012, 19th Conference on Scientific Computing, edited by A. Handlovicova, Z. Minarechova, D. Sevcovic (Vysoke Tatry - Podbanske, Slovakia, 2012), pp. 331 341

9. F. Harlow, J. Welch, Physics of Fluids 8, 2182 (1965)

10. J. Klostermann, K. Schaake, R. Schwarze, International Journal for Numerical Methods in Fluids 71, 960 (2013)

11. J.A. Sethian, Level Set Methods and Fast Marching Methods, Cambridge Monograph on Applied and Computational Mathematics, $2^{\text {nd }}$ edn. (Cambridge University Press, Cambridge, U.K., 1999)

12. S. Basting, M. Weismann, Journal of Computational Physics 255, 228 (2013)

13. M. Raessi, H. Pitsch, Computers \& Fluids 63, 70 (2012)

14. E. Bänsch, Numer. Math. 88, 203 (2001)

15. D. Kuzmin, Journal of Computational Physics 219, 513 (2006) 